\title{
COMPARATIVE STUDY OF ARTHROCENTESIS WITH OR WITHOUT USING PIROXICAM IN THE MANAGEMENT OF TEMPOROMANDIBULAR JOINT DISORDERS
}

\author{
AL-Said S N ${ }^{1} B D S$, Shawky $\mathrm{N}^{2} P h D$, Ragab $\mathrm{H} \mathrm{R}^{3} P h D$.
}

\begin{abstract}
: thereby, relieving pain.

Objective: To evaluate the efficacy of arthrocentesis with and without the injection of piroxicam on TMD. range of motion, joint effusion and joint sounds. its clinical outcome.

Key Words: Arthocentesis, intra-articlar, injection, temporomandibular joint dysfunction.

1. B.D.S. Faculty of Dentistry, October 6 University.

2. Professor of Oral \& Maxillofacial Surgery, ,Faculty of Dentist, Alexandria University

3. Assistant professor of Oral and Maxillofacial Surgery, Faculty of Dentist, Alexandria University.
\end{abstract}

Introduction: Temporomandibular joint dysfunction (TMD) is a clinically significant condition which can be a source of acute or chronic orofacial pain and dysfunction including limitation of mandibular movement. Arthrocentesis is joint lavage which washes out these inflammatory mediators,

Materials and methods: This study was conducted on twenty patients with clinical and radiological diagnosis of TMD included in the study divided into equal groups. Ten patients were subjected to conventional arthrocentesis with saline solution alone, other ten patients underwent arthrocentesis by injection of piroxicam. Patient's evaluation will be preoperatively and postoperatively following the procedure and after 1 st, 2 nd week, 1 and 6 months. Magnetic resonance imaging (MRI) was performed in both groups before starting the treatment.

Results: Evaluation of assisted and unassisted mouth opening showed increase at all time intervals. There was significant improvement in pain,

Conclusion: Arthrocentesis combined with piroxicam injections is superior to arthrocentesis alone in reducing impairment in patients with TMD on

\section{INTRODUCTION}

The temporomandibular joint (TMJ) presents many problems in modern medicine. Patients who suffer from TMJ disorders often experience a major loss in quality of life due to the debilitating effects that TMJ disorders can have on everyday activities (1).

Temporomandibular disorders (TMDs) have been identified as a major cause of non-dental pain in the orofacial region and are considered to be a subclassification of musculoskeletal disorders (2).

Despite much research into the diagnosis and treatment of TMD, the true nature of the disease remains incompletely understood and a wide variety of therapies are applied (3).

Temporomandibular joint (TMJ) is a compound joint, composing of temporal bone and mandible, numerous associated muscles and a specialised fibrous tissue the articular disk (4).

Anatomically TMJ is a diarthrodial synovial joint, with associated muscles and ligaments, dictating and limiting the freedom of discontinuous articulation between two bones (5).

Temporomandibular joint disorder (TMD) may cause pain, clicking, crepitus and irregular or deviating jaw function. From a clinical point of view, TMJ internal derangement has two expressions: painful clicking and chronic closed lock which is associated with osteoarthritis $(6,7)$.

Cardinal signs of TMJ disorder's (internal derangement) may be (a) limitation of mandibular movement (b) pain with mandibular function (c) joint sounds. Successful treatment depends on accurate assessment, comprehensive evaluation and diagnosis (8).

Various non-surgical and surgical treatment modalities of TMJ disorder's (internal derangement) have been contemporarily described. Arthrocentesis is a simple, safe and minimally invasive technique for the treatment of TMJ disorders. Significant improvements have been reported in terms of reduction in TMJ pain on mouth opening and clicking or popping sounds in the TMJ following arthrocentesis (9-11).

Arthrocentesis is effective for washing out bradykinin, interleukin-6 and protein from the TMJ (12).

Most common intra-articular injections following arthrocentesis are steroids and sodium hyaluronate $(13,14)$.

Non-steroidal anti-inflammatory drugs (NSAIDs) have been used to treat acute and chronic inflammatory articular disorders, such as rheumatoid arthritis and osteoarthritis. Combined treatment with arthrocentesis and NSAIDs for inflamed synovial joint removes the inflammatory mediators, alters the intra-articular pressure and reduces synovial inflammation (15).

Piroxicam is an oxicam NSAID; its plasma half-life has been estimated at 45hours, allowing for once-daily dosing, with peak plasma concentration occurring 2 to 4 hours after oral administration. Piroxicam in single dose of 20 to $40 \mathrm{mg}$ has been shown to produce analgesia with longer duration of action.(16)

Piroxicam has been used for the treatment of rheumatoid arthritis and osteoarthritis. The parenteral formulation of 
piroxicam has an aqueous base, without an organic stabiliser and since the solvent for injection is distilled water, this formulation offers the potential for intra-articular administration. Piroxicam has also been shown to concentrate in the synovium rather than in the cartilage (17).

The aim of this study was to evaluate the efficacy of arthrocentesis with and without the injection of piroxicam on TMD.

\section{MATERIALS AND METHODS}

This study was conducted on 20 patients selected from those attending the outpatient clinic in Oral and Maxillofacial Surgery Department, Faculty of Dentistry, Alexandria University between August 2014 and July 2015. The ethical clearance was obtained by the ethical committee before the study began and the selected patients were informed about the nature of the study and informed consents were signed.

Different conservative modalities were used before the arthrocentesis procedure including soft diet, moist heat application, analgesic anti-inflammatory drugs and splint therapy.

\section{Inclusion criteria of patient selection}

- A report of orofacial pain referred to the TMJ.

- Pain and tenderness of the TMJ during mandibular movement.

- Limited mouth opening.

- Clicking of the joint during movement.

- Patient who did not respond to conservative management including splint therapy.

Patients excluded from the study were those:

- Presence of other disorders involving the TMJ (e.g., myalgia, collagen vascular disease).

- Dentofacial deformity.

- Psychiatric illness.

- With previous TMJ surgery.

- Patients suffering from joint trauma, cellulitis or severe laceration of pre-auricular skin covering the TMJ area.

\section{Patients were divided into 2 groups}

- Group I: Contained 10 patients who underwent conventional arthrocentesis (control group).

- Group II: Contained 10 patients who underwent arthrocentesis with injection of piroxicam (study group).

\section{Preoperative phase}

1) Clinical diagnosis

a) Patient questionnaire: all details were recorded in a questionnaire by the examiner including: Chief complaint, Personal data, Past history.

b) Clinical examination: general body examination, evaluation of mandibular range of motion.

2) Occlusal splint therapy fabrication and insertion: hard occlusal splint was constructed and adjusted for each patient of TMD in both groups before arthrocentesis.

3) Magnetic resonance imaging: radiological diagnosis of TMJ Disorder, based on clinical diagnostic criteria was included in the study, MRI was performed in both groups; Position of the disc, disc configuration, osseous abnormalities of the condyle and temporal eminence and effusion. (Fig. 1)

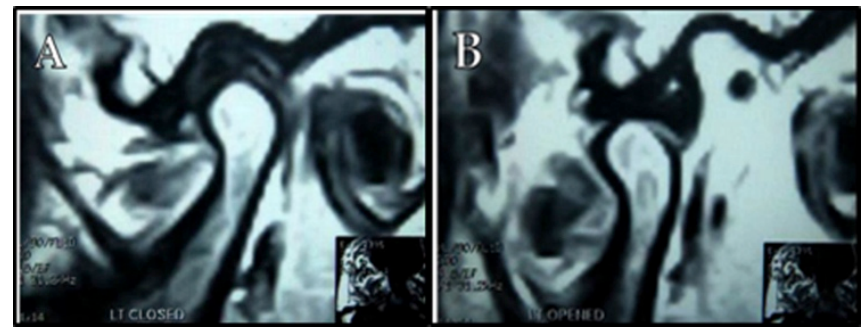

Fig. (1): Oblique sagittal MRI T1-weighted image for the left TMJ. (A) In close open position (B) In open positions showing anterior displacement of the left TMJ disc with reduction.

\section{Operative phase}

In both groups, the same technique of arthrocentesis in the affected joint was performed following a procedure (18-20):

Local anaesthetic (Mepivican with adrenaline, each carpule is $1.8 \mathrm{ml}$. Mepevacaine HCL 2\%. Produced by: Alexandria Co. for pharmaceuticals, Alexandria, Egypt) block of auriculotemporal nerve and infiltration into the areas of joint penetration.

Surgical field is draped and painted with Betadine (Betadine: $7.5 / \mathrm{M} / \mathrm{V}$ povidone iodine USP Nile Company, Under License by Switzerland). External auditory canal is protected from accumulation of blood and fluid used a cotton pledget.

A line was drawn from the corner of the eye to midpoint of tragus (canthal-tragus line), the first mark was made $1 \mathrm{~cm}$ from the tragus, and the second mark was made 2 mm below. (Fig. 2)

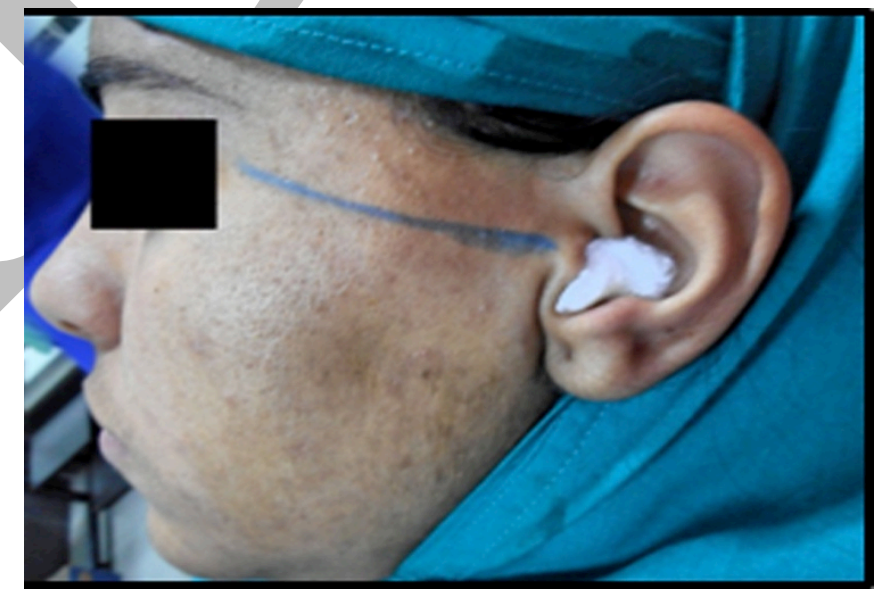

Fig. (2): Showing canthal-tragus line.

A20-gauge needle was introduced reaching the posterior aspect of the articular eminence, approximately 2 $\mathrm{ml}$ of normal saline was injected passively into the joint until there was rebound of syringe with mandibular movement.

A18-gauge needle was then introduced into the second mark (outlet needle), where its tip meets the inlet needle. (Fig. 3)

A total amount of $200 \mathrm{ml}$ of normal saline solution was first introduced into the inlet needle; joint is manipulated 
through opening, closing, protrusive and lateral excursions of the mandible to establish free flow of the solution and release adhesions; then collected into a kidney dish through the outlet needle, inlet needles were inserted to a depth of about $1.5 \mathrm{~cm}$. (Fig. 4)

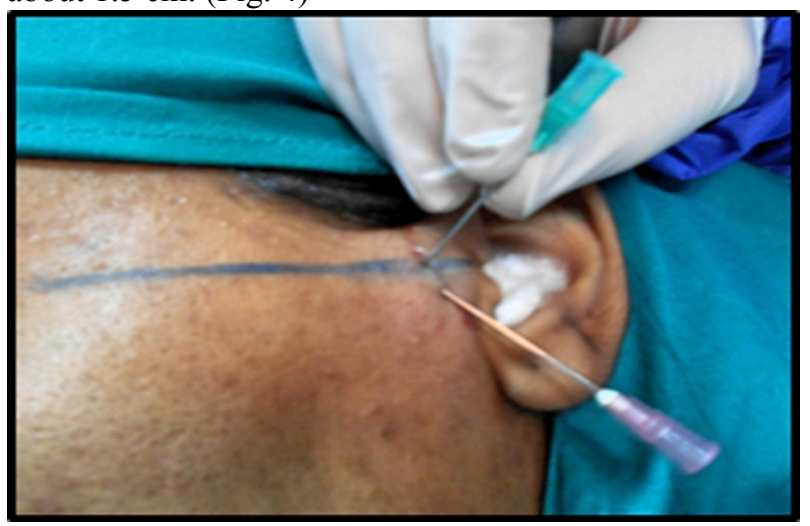

Fig. (3): Showing insertion of inlet and outlet needle.

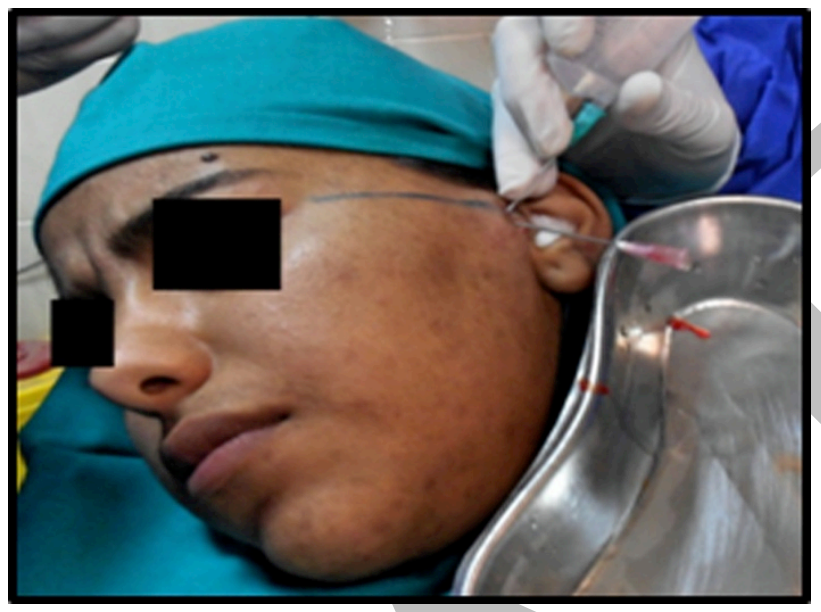

Fig. (4): Showing arthrocentesis.

The procedure of superior joint lavage took from 15-20 minutes. Gentle finger pressure was done over the skin area where the two needles met to help holding the two needles together and to facilitate the outlet flow. During the procedure, the outlet needle was momentarily blocked with finger pressure 2-3 times to help distend and break up the joint adhesion and in group II at the end of procedure the outflow needle was removed and first needle was used to inject $2 \mathrm{ml}(40 \mathrm{mg})$ of piroxicam (Feldene: $1 \mathrm{ml}$ ampoule piroxicam manufactured by Pfizer S.A.E) into the joint spac. (Fig. 5)

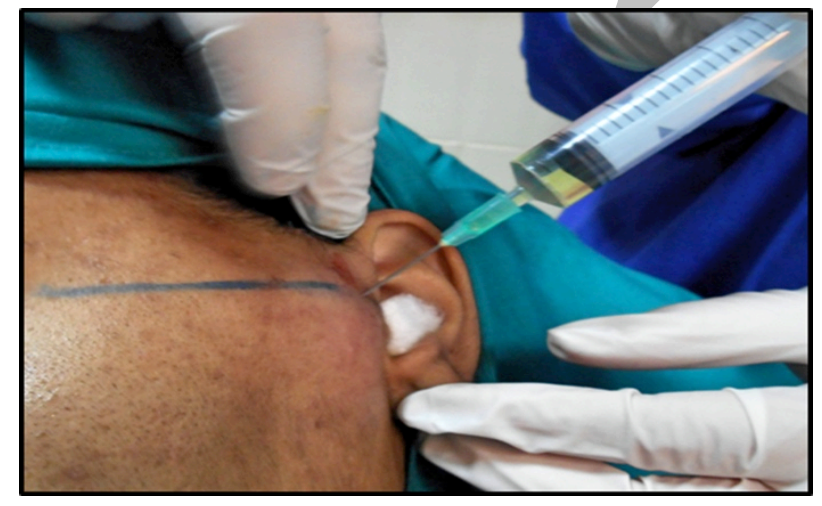

Alexandria Dental Journal. (2015) Vol.40 Pages:160-165
Fig. (5): Showing Injection of piroxicam.

After removing the needles, the jaw was gently manipulated in vertical, protrusive and lateral excursions.

\section{Post-operative phase}

After the arthrocentesis, for all the patients:

Postoperative medication were prescribed consisting of an antibiotic, Flumox $500 \mathrm{mg}$ (Each capsule contains: 250 $\mathrm{mg}$ Amoxycillin (as trihydrate) and $250 \mathrm{mg}$ Flucloxacillin (as monohydrate), produced by: Egyptian Int. Pharmaceutical Industries Co. E.I.P.I.CO. 10th of Ramadan City, Area B1 P.O. Box 149 Tenth, Egypt), to be taken 3 times daily for 5 days to protect against any possibility of infection.

Postoperative instructions were given regarding soft diet and home physical therapy consisting of the application of moist heat and mandibular exercises (consisting of mouth opening, protrusion and lateral movements) 4 times daily.

Both groups of patients were instructed to wear their splints during the daytime and at night during sleep for one month.

\section{Follow-up}

Follow-up was done at 24 hours, 1 week, 2 week, 1 month, 6 months post operatively.

Postoperative evaluation included the following parameters:

- $\quad$ Pain level using Visual Analogue Scale (VAS I).

- Maximum Inter Incisal Opening (MIO).

- Range of lateral and protrusive movement.

- Presence of joint noise on opening and closing.

- Tenderness of joint.

Statistical analysis of Qualitative data were described using range (minimum and maximum), mean, standard deviation and median. Comparison between different groups regarding categorical variables was tested using Chi-square test and Fisher's Exact test, While, McNemar test was used to analyze the significance between the different stages of time. The distributions of quantitative variables were tested for normality using Kolmogorov-Smirnov test, ShapiroWilk test and D'Agstino test, if it reveals normal data distribution, parametric tests was applied. Comparison between two independent population were done using independent t-test, also paired t-test is used to analyses two paired data. For abnormally distributed data, comparison between two independent population were done using Mann Whitney test, To compare between the different periods Wilcoxon signed ranks test was applied.

\section{RESULTS}

The patients were selected from those attending the outpatient clinic in Oral and Maxillofacial Surgery Department, Faculty of Dentistry, Alexandria University. Patients' ages ranged from 18-50 years (with a mean age range of 29.6) from both genders (male and female) with duration of presenting symptoms 5 months to 3 years. Regarding the gender, seventeen patients were females and three patients were males. 
All patients were diagnosed with TMD (internal derangement) of different stages from early to late. Diagnosis of internal derangement and TMD was based on clinical data and magnetic resonance imaging findings.

Control group included: ten patients they had the following MRI findings; Anterior disc displacement with reduction six joints; Anterior disc displacement without reduction 4 joints.

Study group: included ten patients were follows MRI findings; anterior disc displacement with reduction 7 joints, anterior disc displacement without reduction 3 joints.

Every case was monitored on intervals of $24 \mathrm{hrs}, 1$ week, 2 week, 1 month and 6 month, to evaluate pain intensity, maximal interincisal opening, lateral and protrusive movements, clicking, and joint tenderness. No complains or complications were seen following the arthrocentesis or injection of piroxicam.

The statistical analysis of pain intensity table (1) data showed significant decrease between the preoperative pain score comparing with $24 \mathrm{hrs}, 1$ week, 2 week, 1 month and 6 month in group I and group II while comparing the two groups there was no statistically significant difference in the pain intensity between both groups during intervals period $(\mathrm{p}>0.05)$.

Table (1): Comparison between the two study groups according to pain intensity.

\begin{tabular}{|c|c|c|c|c|c|c|}
\hline & \multicolumn{6}{|c|}{ Pain intensity } \\
\hline & $\begin{array}{c}\text { Pre- } \\
\text { operative }\end{array}$ & 24 hours & 1 Week & 2 Week & 1 Month & 6 Month \\
\hline \multicolumn{7}{|l|}{ Control $(n=10)$} \\
\hline Min. - Max. & $6.0-9.0$ & $3.0-7.0$ & $1.0-5.0$ & $1.0-3.0$ & $0.0-3.0$ & $0.0-5.0$ \\
\hline Mean \pm SD. & $7.60 \pm 0.97$ & $4.70 \pm 1.42$ & $3.10 \pm 1.37$ & $1.90 \pm 0.74$ & $1.60 \pm 0.97$ & $2.60 \pm 1.78$ \\
\hline Median & 7.50 & 4.50 & 3.0 & 2.0 & 1.50 & 2.50 \\
\hline $\mathbf{p}_{1}$ & & $0.010^{\circ}$ & $0.005^{*}$ & $0.004^{\circ}$ & $0.005^{*}$ & $0.005^{*}$ \\
\hline \multicolumn{7}{|l|}{ Study $(n=10)$} \\
\hline Min. - Max. & $6.0-9.0$ & $1.0-5.0$ & $0.0-4.0$ & $0.0-3.0$ & $0.0-2.0$ & $0.0-4.0$ \\
\hline Mean \pm SD. & $7.50 \pm 1.08$ & $3.40 \pm 1.26$ & $2.10 \pm 1.37$ & $1.30 \pm 1.16$ & $1.0 \pm 1.05$ & $1.70 \pm 1.57$ \\
\hline Median & 7.50 & 3.50 & 2.0 & 1.0 & 1.0 & 1.50 \\
\hline $\mathbf{p}_{1}$ & & $0.005^{\circ}$ & $0.005^{*}$ & $0.005^{\circ}$ & $0.005^{*}$ & $0.005^{*}$ \\
\hline $\mathbf{z}$ & 0.197 & 1.829 & 1.454 & 1.300 & 1.194 & 1.156 \\
\hline p & 0.844 & 0.067 & 0.146 & 0.194 & 0.233 & 0.248 \\
\hline
\end{tabular}

Z: Z for Mann Whitney test

$\mathrm{p}$ : $\mathrm{p}$ value for Wilcoxon singned ranks test for comparing between Pre-operative with other periods

*: Statistically significant

Regarding the maximum mouth opening, a limited mouth opening was obvious preoperatively but the mouth opening (MO) improved postoperatively. The maximum mouth opening scores at $24 \mathrm{hrs,} 1$ week, 2 week, 1 month and 6 month is show in table (2). The mouth opening was increased in both groups throughout the following up periods no statistically significant difference in the MO between both groups during follow up period ( $p>0.05$ ).

The lateral movement (towards the unaffected side) was measured in groups I \& II. They were increased in group I throughout the follow up periods 1 week, 2 weeks and 1 month comparing between pre-operative with each period while in group II increased during following up periods 24 hours, 1 week and 2 weeks. Comparing the two groups there was no statistically significant difference in the lateral movement between both groups during the follow up period $(\mathrm{p}>0.05)$.

Table (2): Comparison between the two studied groups according to maximal interincisal opening.

\begin{tabular}{|c|c|c|c|c|c|c|}
\hline & \multicolumn{6}{|c|}{ Maximal interincisal opening (MIO) } \\
\hline & $\begin{array}{c}\text { Pre- } \\
\text { operative }\end{array}$ & 24 hours & 1 Week & 2 Week & 1 Month & 6 Month \\
\hline \multicolumn{7}{|l|}{ Control $(n=10)$} \\
\hline Min. - Max. & $19.0-25.0$ & $26.0-37.0$ & $31.0-36.0$ & $28.0-38.0$ & $32.0-35.0$ & $26.0-36.0$ \\
\hline Mean $\pm \mathrm{SD}$. & $\begin{array}{c}21.20 \pm \\
2.25\end{array}$ & $\begin{array}{c}31.14 \pm \\
4.11\end{array}$ & $34.10 \pm 1.91$ & $33.6 \pm 4.09$ & $33.80 \pm 1.23$ & $\begin{array}{c}31.54 \pm \\
3.97\end{array}$ \\
\hline Median & 20.0 & 30. & 34.50 & 36.0 & 34.0 & 32.0 \\
\hline $\mathbf{p}_{1}$ & & $<0.001^{*}$ & $<0.001^{*}$ & $<0.001^{*}$ & $<0.001^{*}$ & $<0.001^{*}$ \\
\hline \multicolumn{7}{|l|}{ Study $(n=10)$} \\
\hline Min. - Max. & $19.0-26.0$ & $28.0-40.0$ & $29.70-42.0$ & $31.50-43.0$ & $31.50-43.0$ & $29.30-4.0$ \\
\hline Mean \pm SD & $\begin{array}{c}21.48 \pm \\
1.95\end{array}$ & $\begin{array}{c}34.90 \pm \\
4.37\end{array}$ & $36.48 \pm 3.19$ & $36.79 \pm 3.63$ & $36.44 \pm 3.95$ & $\begin{array}{c}35.12 \pm \\
3.74\end{array}$ \\
\hline Median & 21.0 & 37.0 & 36.20 & 36.50 & 37.0 & 35.20 \\
\hline $\mathrm{p}_{1}$ & & $<0.001^{\circ}$ & $<0.001^{*}$ & $<0.001^{*}$ & $<0.001^{*}$ & $<0.001^{\circ}$ \\
\hline $\mathrm{t}$ & 0.297 & 1.981 & 2.025 & 1.844 & 2.016 & 2.077 \\
\hline p & 0.770 & 0.063 & 0.058 & 0.082 & 0.070 & 0.052 \\
\hline
\end{tabular}

t: Student t-test

$\mathrm{p}$ : $\mathrm{p}$ value for Paired t-test for comparing between Pre-operative with other periods

*: Statistically significant

The increase in protrusive movement from preoperative to $24 \mathrm{hrs}$, 1 week, 2 week, 1 month and 6 month postoperative was found to be statistically significant in group I and also in group II. Regarding protrusive movement, the difference between the two groups throughout the whole follow-up period was found to be statistically insignificant $(p>0.05)$.

Regarding to TMJ clicking in group I before arthrocentesis six of the ten patients $(60 \%)$ had detectable joint sounds. By the end of the six months follow-up period 6 patients were still complaining of clicking while In group II before arthrocentesis seven of the ten patients (70\%) had detectable joint sounds, this decreased to three patient out of ten $(30 \%)$ by the end of the six months follow-up period. There was no statistically significant difference between the two groups regarding TMJ clicking preoperatively and during the postoperative follow- up period.

The group I \& II patients had joint tenderness preoperatively. After 24 hours postoperative all patients had joint tenderness in group I and six patients had joint tenderness in group II. The difference between the two groups throughout the whole follow-up period of joint tenderness was found to be statistically insignificant.

\section{DISCUSSION}

Temporomandibular joint disorder (TMD) refers to a complex and poorly understood set of conditions, manifested by pain in the area of jaw and limitation in the ability to make normal movements of speech, eating, chewing and swallowing. TMD occurs when the muscles used in chewing and the joints of the jaw fail to work in combination with each other. So that TMD is an umbrella term which combines those with the true pathology of the 
TMJ and those with the involvement of the muscles of mastication. These symptoms are interrelated. Increased pain causes decreased maximal opening, which in turn causes increased dysfunction. Thus, correction of one problem can lead to correction of the other two $(21,22)$.

In this study twenty patients were selected, seventeen patients were female and three patients were male. This sample goes in agreement with several studies that concluded that women had a significantly higher prevalence of clinical signs. They reported that female sex seemed to be an increased risk factor for TMD pain (23-27).

Arthrocentesis was performed in the present study under local anesthesia, sterile normal saline followed by injected piroxicam in group II (Study group) and sterile normal saline as lavage solution in group I (Control group).

Arthrocentesis was done only on the affected side. Clinically the results of the present study revealed a significant increase in the measurements of mandibular movements including maximal interincisal opening, lateral and protrusive movement. The finding of improvement in mandibular range of motion was in accordance with Nitzan et al (26) who reported that arthrocentesis proved to be highly effective, providing significant improvement in maximal mouth opening and lateral movement and protrusive movement in all patients.

The results of the present study showed a significant reduction in pain intensity after arthrocentesis. This finding is in agreement with the results obtained by several authors $(11,28,29)$ who reported an improvement in the pain level post arthrocentesis in their studies by washing out of inflammatory mediators by arthrocentesis which had its effect in pain reduction and increasing range of movement. The amount of pain decrease was greater in group II which piroxicam used after sterile lavage solution after 24 hours, differences between the two groups were statistically nonsignificant at several time points of the postoperative period.

Internal derangement (ID) is often associated with inflammation of joint space and on physical examination, a clicking sound is heard on opening or closing of the jaw, with associated pain. The popping is due to the noise that the condyle makes as it moves under the anteriorly displaced disc (30).

The procedure of lysis and lavage was widely accepted to have minimal risks (31). On the other hand, some postoperative complication were record in this study, eight patients have pre-auricular swelling due to extra-articular fluid extravasation which resolved by second postoperative day. Wherefore, four patients reported difficult closure of the eyelids may due to transient facial anesthesia and resolved after two hours postoperatively.

\section{CONCLUSIONS}

From the results of this study we can conclude that:

- Conservative therapy was very important as initial treatment of TMD.

- Arthrocentesis are recommended as a simple alternative to more invasive temporomandibular joint procedures and as an effective technique for the treatment of temporomandibular disorders.

- The washing out of inflammatory mediators by arthrocentesis, as well as the direct action of instilled medications on intracapsular receptors have their effect in pain reduction and increasing range of movement.

- Piroxicam has an anti-inflammatory effect, which explains the fast effect on pain relief, it also improves and restores normal lubrication of the joint.

- Intra-articular injection of piroxicam at the end of arthrocentesis procedure did not show significant effect on the postoperative pain.

\section{CONFLICT OF INTEREST}

The authors declare that they have no conflicts of interest.

\section{REFERENCES}

1. Choi BH, Yoo JH, Lee WY. Comparison of magnetic resonance imaging before and after non surgical treatment of closed lock. Oral Surg Oral Med Oral Pathol 1994; 78: 301-5.

2. Bailey MM, Wang L, Bode CJ, Mitchell KE, Detamore MS. A comparison of human umbilical cord matrix stem cells and temporomandibular joint condylar chondrocytes for tissue engineering temporomandibular joint condylar cartilage. Tissue Eng 2007; 13: 2003-10.

3. Okeson JP. Bell's Orofacial Pains. 5th ed. Chicago: Quintessence, 1995. P 123-33.

4. Yeung RWK, Xia JJ, Samman N. Image-guided minimally invasive surgical access to the temporomandibular joint: A preliminary report. J Oral Maxillofac Surg 2006; 64: 1546-52.

5. Miloro M, Ghali GE, Peter EL, Peter DW. Peterson's Principles of Oral and Maxillofacial Surgery. 2nd ed. London: BC Decker Inc Hamilton, 2004. P 963-89.

6. Wadhwa S, Kapila S. TMJ disorders: future innovations in diagnostics and therapeutics. J Dent Educ 2008; 72: 930-47.

7. Examination, diagnosis, management TMJ disorders: a report of the president's conference. J Am Dent Assoc 1988; 66: 75.

Peagle D, Holmund A, Hjerpe A. Expression of peotoglycan mRNA in patients with painful clinking and chronic closed lock of TMJ. Int J Oral maxillofac Surg 2005; 34: 656-8.

9. Fonseca R, Marciani R, Turvey T. Oral and maxillofacial surgery. 2nd ed. St. Louis (MO): Saunders, Elsevier, 2009. P 815, 833.

10. Hosaka H, Murakami K, Goto K, Iizuka T. Outcome of arthrocentesis for temporomandibular joint with closed lock at 3 years follow-up. Oral Surg Oral Med Oral Pathol Oral Radiol Endod 1996; 82: 501-4.

11. Nishimura M, Segami N, Kaneyama K, Suzuki T. Prognostic factors in arthrocentesis of the temporomandibular joint: Evaluation of 100 patients with internal derangement. J Oral Maxillofac Surg 2001; 59: 874-7.

12. Nitzan DW, Samson B, Better H. Long-term outcome of arthrocentesis for sudden-onset, persistent, severe closed lock of the temporomandibular joint. J Oral Maxillofac Surg 1997; 55: 151-7.

13. Kaneyama K, Segami N, Nishimura M, Sato J, 
Fujimura K, Yoshimura H. The Ideal lavage volume of Bradykinin, Interleukin 6 and protein from the temporomandibular Joint by arthrocentesis. J Oral Maxillofac Surg 2004; 62: 657-61.

14. Al-Belasy FA, Dolwick MF. Arthrocentesis for the treatment of temporomandibular joint closed lock: A review article. Int J Oral Maxillofac Surg 2007; 36: 773-82.

15. Ethunandan M, Wilson AW. Temporomandibular joint arthrocentesis-more questions than answers? J Oral Maxillofac Surg 2006; 64: 952-5.

16. Ishimaru JI, Ogi N, Mizui T, Miyamoto K, Shibata T, Kurita K. Effects of a single arthrocentesis and a COX2 inhibitor on disorders of temporomandibular joints. A preliminary clinical study. Br J Oral Maxillofac Surg 2003; 41: 323-8.

17. Dionne RA, Berthold CW. Therapeutic uses of nonsteroid anti-inflammatory drug in dentistry. Crit Rev Oral Biol Med 2001; 12: 315-30.

18. Gert S, Hans K, Josef S. MRI imaging of the TMJ: MRI diagnosis and intraoperative findings. J Cranio Maxillofac Surg 1993; 21: 284-8.

19. Rüdiger E, Stefan G, Thomas E. Magnetic resonance imaging findings of internal derangement, osteoarthrosis, effusion and bone marrow edema before and after performance of arthrocentesis and hydraulic distension of the temporomandibular joint. Oral Surg Oral Med Oral Pathol 2006; 101: 784-90.

20. William C, Marianne P, Lewis R. A modified auriculotemporal nerve block for regional anesthesia of the temporomandibular joint. J Oral Maxillofac Surg 1984; 42: 544-5.

21. Carvajal W, Laskin DM. Long-term evaluation of arthracentesis for the treatment of internal derangements of the temporomandibular joint. J Oral Maxillofac Surg 2000; 58: 852-5.

22. Venancio RA, Camparis CM, Lizarelli RZ. Low intensity laser therapy in the treatment of temporomandibular disorders: a double-blind study. J Oral Rehabilitation 2005; 32: 800-7.

23. Abd-Ul-Salam H, Weinberg S, Kryshtalskyj B. The incidence of reoperation after temporomandibular joint arthroscopic surgery: a retrospective study of 450 consecutive joints. Oral Surg Oral Med Oral Pathol Oral Radiol Endod 2002; 93: 408-11.

24. Matsuka Y, Yatani H, Kuboki T, Yamashita A. Temporomandibular disorders in the adult population of Okayama City, Japan. Cranio 1996; 14: 158-62.

25. Gesch D, Bernhardt O, Alte D, Schwahn C, Kocher T, John U, et al. Prevalence of signs and symptoms of temporomandibular disorders in an urban and rural German population: results of a population-based Study of Health in Pomerania. Quintessence Int 2004; 35: $143-50$.

26. Nitzan DW, Dolwick MF, Martinez GA. Temporomandibular joint arthrocentesis: A simplified treatment for severe, limited mouth opening. J Oral Maxillofac Surg 1991; 49: 1163-7.

27. Guo C, Shi Z, Revington P. Arthrocentesis and lavage for treating temporomandibular joint disorders.
Cochrane Database Syst Rev 2009; 4: CD004973.

28. Abdel Hamid HA. Evaluation of the effectiveness of low intensity laser therapy after arthrocentesis in treatment of temporomandibular joint disorders. M.Sc. Thesis. Alexandria University. 2010.

29. Nabil L. Clinical Assessment of Arthrocentesis using ozonized water with sodium hyaluronate injection in management of TMJ Disorders. Master Thesis. Alexandria University. 2008.

30. Upton LG, Wijeyesakere SJ. The incidence of tinnitus in people with disorders of the temporomandibular joint. Int Tinnitus J 2004; 10: 174-6.

31. Kaneyama K, Segami N, Nishimura M, Suzuki T, Sato J. Importance of proinflammatory cytokines in synovial fluid from 121 joints with temporomandibular disorders. Br J Oral Maxillofacial Surgery 2002; 40: 418-23. 